\title{
Assessing Organic Manure Efficiency Versus Mineral Fertilization with and without Mycorrhiza on Yield and Properties of Globe Artichoke (Cynara scolymus L.) Grown on a Clay Alluvial Soil in Egypt Delta \\ Nahla M. Morsy
}

Department of Sustainable Development of Environment and it's projects Management,

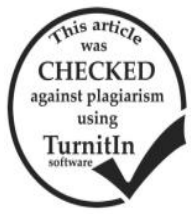
Environmental Studies \& Research Institute (ESRI), EL-Sadat City University, Egypt.

E-mail address: Nahla.morsy@esri.usc.edu.eg

\begin{abstract}
A field experiment was conducted for two successive seasons (2015/2016 and 2016/2017) on globe artichoke (Cynara scolymus L.) cv. Fransawy in a randomized complete block, factorial. Factor 1 was manuring (M) treatments of mineral nutrients " as a reference treatment" "RT" (M1), farmyard manure "FYM" (M2), vermicompost "VC" (M3), chicken manure "CM" (M4) and biogas compost "BC" (M5). Factor 2 was Arbuscular mycorrhiza (A) with treatments of none (A0) and mycorrhiza (A1). Mineral fertilizations (kg ha $\left.{ }^{-1}\right)$ were $124 \mathrm{~N}+65 \mathrm{P}+200 \mathrm{~K}$. Organic fertilization was on basis of applying $124 \mathrm{~kg}$ total $\mathrm{N} \mathrm{ha} \mathrm{a}^{-1}$ of each source with the associated $\mathrm{P}$ and $\mathrm{K}$ in each. Rates of sources $\left(\mathrm{kg} \mathrm{ha}^{-1}\right)$ were 34972.1 FYM, 4124.6 CM, 6887.9 BC and 7614.9 VC (as an average in both seasons). RT gave greater values of number of leaves, number of off-shoots, plant height as well as NPK contents and yield than any of the organic manures with relative yield production efficiency of 80.9, 76.7, 68.2 and 66.7 \% for VC, CM, FYM and BC respectively relative to RT. Vesicular Arbuscular Mycorrhizal (VAM) caused increases in all parameters only under conditions of organic manuring. Keywords: artichoke, mycorrhiza, farmyard manure, vermicompost, chicken manure, biogas, productivity
\end{abstract}

\section{INTRODUCTION}

Globe artichoke (Cynara scolymus L.) is an important vegetable crop in Egypt (Shams, 2014). Its immature inflorescence (head) is the edible part (Rouphael et al., 2017). It is rich in inulin and vitamins (Frutos et al., 2019), fibers, minerals (Lattanzio et al., 2009), polyphenols (Fratianni et al., 2007) and antioxidants (Liorach et al, 2002). Thus, it gained a high importance for export to the European markets (Barno et al., 2011).

Although inorganic soluble fertilizers are commonly used for vegetable crops (Lampkin, 1990) in view of their quick utilization by plants (Vernon, 1999; Duhan et al., 2017). However, the extensive fertilization contributed in environmental pollution (Abdelhafez et al., 2012) besides their high cost of application (Horton and Manner, 2018). On the other hand, biofertilizers and organic amendments and fertilizers can be used as environment-friendly sources for plant nutrients although they may not be as efficient in crop production as chemical fertilizers (Farid et al., 2014; Abdelhafez et al., 2017; Alshaal et al., 2019; Bassouny and Abbas, 2019; Wakindiki et al., 2019). Organic manures such as compost are thought to improve the soil structure, aeration and support plants with slow release nutrient (Uddin et al., 2009); however, the immature compost might have negative impacts on soils and plants (Duggan and Jones, 2016). Composting using worms is also thought to be a suitable alternative (Kumar et al., 2015). This product has lower $\mathrm{pH}$ value and narrower $\mathrm{C} / \mathrm{N}$ ratio as compared with the compost (Duggan and Jones, 2016). Biogas is another organic amendment that can increase soil fertility; hence improve the growth of plants (Farid et al., 2018). Farmyard manure can increase enrich soil with nutrients (Nest et al., 2016). All such organic amendments can enrich soil with nutrients (Abdelhafez et al., 2018; Mupambwa and
Mnkeni, 2018) and stimulate the microbial biomass activities in soil (Sradnick et al., 2018). Mixed applications of these amendments can be more efficient in improving plant growth and yield (Farid et al., 2018).

Phosphorus (P) affects plant productivity particularly in soil where its fixation occurs easily, such as many soils in Egypt (El-Katkat, 1992). Organic amendments can release $\mathrm{P}$ slowly upon their decomposition (Ahmed et al., 2013). However, application of the organic treatments with no other amendments might not be the optimum solution to improve the availability of $\mathrm{P}$. In this concern, mycorrhiza is one of the promising biotechniques that can improve $\mathrm{P}$ uptake by plants (Ardakani et al, 2011 and Mohamed et al., 2019) such as artichokes (Ezz El-Din et al, 2010). This takes place through different mechanisms such as solubilizing soil P (Rai, 2006). Extended mycorrhiza hyphae can penetrate soil and increasing the absorption area of the plant roots (Wiedenhoeft, 2006). Applying VAM can increase N utilization by plants (Verzeaux et al., 2017; Vadeboncoeur et al., 2015 and Zhang et al., 2018). The current study is aiming at assessing the effect of different organic amendments vermicompost, chicken manure, biogas compost or farmyard manure applications with or without VAM on yield and properties of Globe artichoke grown on a clay alluvial soil in Egypt Delta.

\section{MATERIALS AND METHODS}

\section{Materials:}

Both soils of the experimental fields were clay nonsaline slightly alkaline alluvial soils; the main properties of the soils are given in Table 1. The manures and VAM fungi (Glomus mosseae) were obtained from Ain Shams University, Faculty of Agriculture. The main properties of the manures are given in Table 2.

Table 1. Main properties of soils of the fields of the experiment

\begin{tabular}{|c|c|c|c|c|c|c|c|c|c|c|c|}
\hline \multirow{2}{*}{ Season } & Sand & Silt & Clay & \multirow{2}{*}{ Texture } & \multirow{2}{*}{ pH } & \multirow{2}{*}{$\begin{array}{c}E C \\
\left(\mathrm{dS} \mathrm{m}^{-1}\right)\end{array}$} & O.M & $\mathrm{CaCO}_{3}$ & $\mathbf{N}$ & P\% & $\mathbf{K}$ \\
\hline & & $\%$ & & & & & \multicolumn{5}{|c|}{$\mathbf{g k g}^{-1}$} \\
\hline 2015 & 21.06 & 25.16 & 53.78 & Clay & 8.2 & 1.03 & 10.1 & 153 & 1.2 & 3.1 & 1.1 \\
\hline 2016 & 21.25 & 26.21 & 52.54 & Clay & 8.5 & 0.95 & 11.3 & 144 & 1.4 & 4.2 & 1.3 \\
\hline
\end{tabular}

pH in soil:water suspension (1:2.5), EC in soil paste extract 
Table 2. Main properties of organic manures used in the experiment

\begin{tabular}{|c|c|c|c|c|c|c|c|c|}
\hline \multirow[b]{2}{*}{ Organic manure } & \multicolumn{4}{|c|}{ Season 1} & \multicolumn{4}{|c|}{ Season 2} \\
\hline & $\begin{array}{c}\text { Farmyard } \\
\text { manure } \\
\text { (FYM) }\end{array}$ & $\begin{array}{c}\text { Chicken } \\
\text { manure } \\
(\mathrm{CM})\end{array}$ & $\begin{array}{c}\text { Biogas } \\
\text { manure } \\
\text { (BC) }\end{array}$ & $\begin{array}{c}\text { Vermi- } \\
\text { compost } \\
\text { (VC) }\end{array}$ & FYM & $\begin{array}{c}\text { Chicken } \\
\text { manure } \\
(\text { CM) }\end{array}$ & $\begin{array}{c}\text { Biogas } \\
\text { manure } \\
(\mathrm{BC})\end{array}$ & $\begin{array}{c}\text { Vermi- } \\
\text { compost } \\
\text { (VC) }\end{array}$ \\
\hline Total N $\left(\mathrm{g} \mathrm{kg}^{-1}\right)$ & 3.0 & 31 & 19 & 15.7 & 4.3 & 29.0 & 17.0 & 16.8 \\
\hline Total P $\left(\mathrm{g} \mathrm{kg}^{-1}\right)$ & 6.5 & 9.4 & 7.5 & 12.7 & 7.0 & 9.2 & 7.0 & 12.3 \\
\hline Total K $\left(\mathrm{g} \mathrm{kg}^{-1}\right)$ & 5.5 & 10.0 & 6.0 & 5.9 & 5.7 & 9.0 & 5.8 & 5.7 \\
\hline Organic matter $\left(\mathrm{g} \mathrm{kg}^{-1}\right)$ & 124 & 406 & 448 & 432 & 101 & 357 & 488 & 401 \\
\hline $\mathrm{C} / \mathrm{N}$ ratio & $25: 1$ & $8: 8$ & $10: 3$ & $10: 7$ & $23: 1$ & $9: 1$ & $10: 5$ & $11: 1$ \\
\hline Density $\mathrm{kg} \mathrm{m}^{-3}$ & 750 & 200 & 285 & 715 & 760 & 220 & 285 & 710 \\
\hline $\begin{array}{l}\text { Organic manure }\left(\mathrm{kg} \mathrm{ha}^{-1}\right) \\
=123.6 \mathrm{~kg} \mathrm{~N} / \mathrm{ha}\end{array}$ & 41200.0 & 3987.1 & 6505.3 & 7872.6 & 28744.2 & 4262.1 & 7270.6 & 7357.1 \\
\hline
\end{tabular}

\section{The Field experiments:}

A field experiment was carried out during the two successive seasons of 2015/2016 and 2016/2017 (one experiment for each season) in a private farm at Damalo village, Benha, Qalubia Governorate. Old crowns of globe artichoke (Cynara scolymus L.) cv. Fransawy (taken from the previous plants) were used as plant materials for propagation. The design was a randomized complete block, factorial (2 factors) with 3 replicates. Factor 1 was manuring (M) with the following treatments: mineral nutrients treatment " as a reference treatment" "RT" $\left(\mathrm{M}_{1}\right)$, farmyard manure "FYM" $\left(\mathrm{M}_{2}\right)$, vermicompost "VC" $\left(\mathrm{M}_{3}\right)$, chicken manure "CM" $\left(\mathrm{M}_{4}\right)$ and biogas compost "BC" $\left(\mathrm{M}_{5}\right)$ respectively. Factor 2 was Arbuscular mycorrhiza $(\mathrm{A})$ with treatments of none $\left(\mathrm{A}_{0}\right)$ and mycorrhiza $\left(\mathrm{A}_{1}\right)$. Plants were planted at end of August. The plot included four ridges, $1 \mathrm{~m}$ wide and $4.0 \mathrm{~m}$ long with an area of $16 \mathrm{~m}^{2}$. Three ridges were planted while the fourth was left without planting as a guard between plots. The old crowns were planted at a distance of $1 \mathrm{~m}$ apart on one side. The reference treatment was given mineral $\mathrm{N}$ at $124 \mathrm{~kg} \mathrm{~N}^{-1}$ as ammonium sulphate $\left(206 \mathrm{~g} \mathrm{~N} \mathrm{~kg}^{-1}\right)+65 \mathrm{~kg} \mathrm{P} \mathrm{ha}^{-1}$ as calcium superphosphate $\left(68 \mathrm{~g} \mathrm{P} \mathrm{kg}^{-1}\right)+200 \mathrm{~kg} \mathrm{~K} \mathrm{ha}^{-1}$ as Ksulphate $\left(400 \mathrm{~g} \mathrm{~K} \mathrm{~kg}^{-1}\right)$. Each of the organic manure treatments were given the manure so as to provide a total of $124 \mathrm{~kg} \mathrm{Nha}^{-1}$. Rates of organic manure applications "kg ha $^{-1}$ " (based on their total N) were thus $34972.1 \mathrm{FYM}$, 4124.6 CM, 6887.9 BC and 7614.9 VC (as an average in both seasons); given during land preparation. Mineral N and $\mathrm{K}$ were given in three equal splits, 2, 3 and 4 months after planting while $\mathrm{P}$ was given with land preparation.

Data recorded:

\section{Vegetative growth characteristics:}

Five plants were randomly taken from each plot for determination of plant height, number of leaves and offshoots per plant at start of blooming (120 days after planting). Samples of leaves were oven dried at $70 \mathrm{C}$ for 48 $\mathrm{h}$, weighed, ground, then digested in a mixture. For determination of $\mathrm{P}$ and $\mathrm{K}$, samples were digested in a mixture of conc. sulphuric and perchloric acids (2:1 ratio), and for $\mathrm{N}$ determination samples were digested with conc. sulphuric acid (Kjeldahl method). Laboratory analyses were carried out according to methods of AOAC (2000) Jackson (1973) and Black et al (1982), respectively.

\section{Head yield and quality:}

Five heads were taken randomly from each plot during every harvest period (early - late) in both seasons of study for measuring the head fresh weight and the average edible part fresh weight. Samples of the edible parts of heads were taken at beginning and end of harvest and dried $70{ }^{\mathrm{O}} \mathrm{C}$. Inulin concentration was determined according to Winton and Winton (1958) while fibers were determined according to AOAC (2000).

\section{Statistical analysis}

Analysis of variance (ANOVA) was performed using the SPSS program (2006). Significant differences between means were determined by Duncan's multiple range test at 5\% according to Gomez and Gomez (1984).

\section{Vegetative growth:}

\section{RESULTS}

Table 3 reveals that application of mineral nutrients (RT) gave greater values of number of leaves, number of off shoots, plant height as well as NPK contents than any of the organic manure treatments. Treatments of the organic manure showed that vermicompost (VC), was the highest followed by chicken manure (CM) then farmyard manure (FYM) and the lowest was by biogas compost (BC). All treatments receiving VAM showed positive response. The positive effect of mycorrhiza occurred only where organic manures were applied, but not where mineral fertilization was given. Therefore, the effect of mineral fertilization was very pronouncing to show any response to the mycorrhiza addition.

\section{Early and total yield:}

Data in Table 4 show that application of the reference treatment (RT) of mineral nutrients combined with VAM gave the highest yields, surpassing the lowest yield given by FYM by 91.7 and $86.6 \%$ for early yield in seasons 1 and 2 respectively. The main effect of fertilization treatments shows that RT gave yields greater than any of the organic manures. The VC manure gave the highest of the organic manures followed by CM then FYM or BC. Relative total yields given by $\mathrm{VC}, \mathrm{CM}, \mathrm{BC}$ and FYM as compared with the RT yield were 80.9, 76.7, 68.2 and $66.7 \%$ respectively (averages of the two seasons). The efficiency of the organic manures was particularly marked under conditions of VAM. The main effect of VAM shows that the mycorrhiza caused an average increase of $23.2 \%$ in early yield over the two seasons. The effect was pronounced under conditions of organic manuring. Where mineral fertilization was given, the VAM effect was very slight. The pattern of response regarding the total yield was rather similar to that of the early yield

\section{Head weight:}

Data in Table 4 show that RT combined with VAM gave the highest head weight surpassing the lowest head weight of the FYM treatment 6.4 and $7.0 \%$ for early yield in seasons 1 and 2 respectively. The main effect of fertilization treatments shows that RT gave weight per head greater than any of the organic manures. Among the organic manures, the VC source gave the highest head weight followed by CM then FYM and BC gave the lowest. 
Table 3. Effect of mineral nutrients and organic manures with and without mycorrhiza on artichoke growth and its $N, P$ and $K$ contents

\begin{tabular}{|c|c|c|c|c|c|c|c|c|c|c|c|c|}
\hline \multirow{3}{*}{$\begin{array}{l}\text { Organic } \\
\text { manuring (M) }\end{array}$} & \multicolumn{12}{|c|}{ Arbuscular mycorrhiza (A) } \\
\hline & \multicolumn{3}{|c|}{ Season 1} & \multicolumn{3}{|c|}{ Season 2} & \multicolumn{3}{|c|}{ Season 1} & \multicolumn{3}{|c|}{ Season 2} \\
\hline & $\mathbf{A}_{0}$ & $\mathbf{A}_{1}$ & Mean & $\mathbf{A}_{0}$ & $\mathbf{A}_{1}$ & Mean & $\mathbf{A}_{0}$ & $\mathbf{A}_{1}$ & Mean & $\mathbf{A}_{0}$ & $\mathbf{A}_{1}$ & Mean \\
\hline & \multicolumn{6}{|c|}{ Number of leaves plant ${ }^{-1}$} & \multicolumn{6}{|c|}{ Plant height $(\mathrm{cm})$} \\
\hline $\mathrm{M}_{1}$ & $36.9 \mathrm{a}$ & $37.6 \mathrm{a}$ & $37.3 \mathrm{~A}$ & $38.5 \mathrm{a}$ & $38.5 \mathrm{a}$ & $38.6 \mathrm{~A}$ & $91.5 \mathrm{ab}$ & $94.2 \mathrm{a}$ & $92.9 \mathrm{~A}$ & $92.2 \mathrm{abc}$ & $94.0 \mathrm{a}$ & $93.1 \mathrm{~A}$ \\
\hline $\mathrm{M}_{2}$ & $23.2 \mathrm{c}$ & $31.6 \mathrm{ab}$ & $27.4 \mathrm{C}$ & $27.6 \mathrm{e}$ & 34.2abcde & $31.0 \mathrm{~B}$ & $71.9 \mathrm{f}$ & 85.4 bcd & $78.6 \mathrm{D}$ & $68.2 \mathrm{e}$ & 85.9abcd & $77.1 \mathrm{C}$ \\
\hline $\mathrm{M}_{3}$ & $28.8 \mathrm{bc}$ & $36.0 \mathrm{a}$ & $32.4 \mathrm{~B}$ & 29.4 bcde & $35.9 \mathrm{ab}$ & $32.6 \mathrm{~B}$ & 83.0 cde & $93.3 \mathrm{a}$ & $88.1 \mathrm{~B}$ & 84.2 bcd & $89.9 \mathrm{abc}$ & 87.1B \\
\hline $\mathrm{M}_{4}$ & $27.8 \mathrm{bc}$ & $33.7 \mathrm{ab}$ & $30.7 \mathrm{BC}$ & 28.9 cde & $35.3 \mathrm{abc}$ & $32.1 \mathrm{~B}$ & $83.0 \mathrm{de}$ & 86.9abcd & $85.0 \mathrm{BC}$ & $82.8 \mathrm{~cd}$ & $89.4 \mathrm{abc}$ & $86.1 \mathrm{~B}$ \\
\hline $\mathrm{M}_{5}$ & $25.4 \mathrm{c}$ & $32.0 \mathrm{ab}$ & $28.7 \mathrm{BC}$ & $28.4 \mathrm{de}$ & 34.4abcd & 31.4B & 77.48 ef & $88.5 \mathrm{abcd}$ & $83.0 \mathrm{C}$ & $79.7 d$ & 89.2abcd & $84.5 \mathrm{~B}$ \\
\hline \multirow[t]{2}{*}{ Mean } & 28.4B & $34.2 \mathrm{~A}$ & & $30.5 \mathrm{~B}$ & $35.7 \mathrm{~A}$ & & $81.4 \mathrm{~B}$ & $89.7 \mathrm{~A}$ & & $81.5 \mathrm{~B}$ & $89.7 \mathrm{~A}$ & \\
\hline & \multicolumn{6}{|c|}{ Number of offshoots plant ${ }^{-1}$} & \multicolumn{6}{|c|}{$\mathrm{N} \%$} \\
\hline $\mathrm{M}_{1}$ & $1.32 \mathrm{abcd}$ & 1.66abcd & $1.49 \mathrm{~A}$ & $2.01 \mathrm{abc}$ & $2.08 \mathrm{ab}$ & $2.04 \mathrm{~A}$ & $1.499 \mathrm{~b}$ & $1.766 \mathrm{a}$ & $1.632 \mathrm{~A}$ & $1.510 \mathrm{~b}$ & $1.664 \mathrm{a}$ & $1.587 \mathrm{~A}$ \\
\hline $\mathrm{M}_{2}$ & $0.39 \mathrm{e}$ & $1.67 \mathrm{abcd}$ & $1.03 \mathrm{~B}$ & $1.01 \mathrm{~d}$ & $1.97 \mathrm{abc}$ & $1.49 \mathrm{~B}$ & $1.059 \mathrm{e}$ & $1.258 \mathrm{~cd}$ & $1.158 \mathrm{D}$ & $1.071 \mathrm{f}$ & $1.295 \mathrm{~cd}$ & $1.183 \mathrm{C}$ \\
\hline $\mathrm{M}_{3}$ & $1.28 \mathrm{bcd}$ & $1.79 \mathrm{ab}$ & $1.54 \mathrm{~A}$ & $2.02 \mathrm{abc}$ & $2.23 \mathrm{a}$ & $2.12 \mathrm{~A}$ & $1.280 \mathrm{~cd}$ & $1.425 \mathrm{~b}$ & $1.352 \mathrm{~B}$ & $1.218 \mathrm{de}$ & $1.359 \mathrm{c}$ & $1.288 \mathrm{~B}$ \\
\hline $\mathrm{M}_{4}$ & $1.12 \mathrm{~cd}$ & $1.92 \mathrm{a}$ & $1.52 \mathrm{~A}$ & $1.84 \mathrm{bc}$ & $2.28 \mathrm{a}$ & $2.06 \mathrm{~A}$ & $1.183 \mathrm{~d}$ & $1.298 \mathrm{c}$ & $1.240 \mathrm{C}$ & $1.150 \mathrm{ef}$ & $1.368 \mathrm{c}$ & $1.259 \mathrm{~B}$ \\
\hline $\mathrm{M}_{5}$ & $1.12 \mathrm{~d}$ & $1.32 \mathrm{abcd}$ & $1.22 \mathrm{AB}$ & $1.64 \mathrm{c}$ & $1.96 \mathrm{abc}$ & $1.80 \mathrm{AB}$ & $1.065 \mathrm{e}$ & $1.229 \mathrm{~cd}$ & $1.147 \mathrm{D}$ & $1.074 \mathrm{f}$ & $1.296 \mathrm{~cd}$ & $1.185 \mathrm{C}$ \\
\hline \multirow[t]{2}{*}{ Mean } & $1.05 \mathrm{~B}$ & $1.67 \mathrm{~A}$ & & $1.70 \mathrm{~B}$ & $2.10 \mathrm{~A}$ & & $1.217 \mathrm{~B}$ & $1.395 \mathrm{~A}$ & & $1.204 \mathrm{~B}$ & $1.396 \mathrm{~A}$ & \\
\hline & \multicolumn{6}{|c|}{$\mathrm{P} \%$} & \multicolumn{6}{|c|}{$\mathrm{K} \%$} \\
\hline $\mathrm{M}_{1}$ & $0.265 \mathrm{def}$ & $0.566 \mathrm{a}$ & $0.415 \mathrm{~A}$ & 0.299 cde & $0.560 \mathrm{a}$ & $0.429 \mathrm{~A}$ & $1.134 \mathrm{de}$ & $2.036 \mathrm{a}$ & $1.585 \mathrm{~A}$ & $1.629 \mathrm{ab}$ & $1.904 \mathrm{a}$ & $1.766 \mathrm{~A}$ \\
\hline $\mathrm{M}_{2}$ & $0.211 \mathrm{f}$ & 0.291 cde & $0.251 \mathrm{D}$ & $0.202 \mathrm{f}$ & $0.314 \mathrm{bcd}$ & $0.258 \mathrm{C}$ & $1.098 \mathrm{e}$ & $1.763 \mathrm{abc}$ & $1.430 \mathrm{~A}$ & $1.315 \mathrm{bc}$ & $1.140 \mathrm{c}$ & $1.227 \mathrm{C}$ \\
\hline $\mathrm{M}_{3}$ & 0.292 cde & $0.383 \mathrm{~b}$ & $0.337 \mathrm{~B}$ & $0.242 \mathrm{def}$ & $0.400 \mathrm{~b}$ & $0.321 \mathrm{~B}$ & $1.359 \mathrm{bcde}$ & $1.779 \mathrm{ab}$ & $1.569 \mathrm{~A}$ & $1.105 \mathrm{c}$ & $1.658 \mathrm{ab}$ & $1.381 \mathrm{BC}$ \\
\hline $\mathrm{M}_{4}$ & $0.246 \mathrm{def}$ & $0.347 \mathrm{bc}$ & $0.296 \mathrm{C}$ & $0.275 \mathrm{def}$ & $0.384 \mathrm{bc}$ & $0.329 \mathrm{~B}$ & $1.315 \mathrm{cde}$ & $1.745 \mathrm{abc}$ & $1.530 \mathrm{~A}$ & $1.372 \mathrm{bc}$ & $1.760 \mathrm{ab}$ & $1.566 \mathrm{AB}$ \\
\hline $\mathrm{M}_{5}$ & 0.224 ef & $0.312 \mathrm{bcd}$ & $0.268 \mathrm{CD}$ & $0.215 \mathrm{ef}$ & $0.323 \mathrm{bcd}$ & $0.269 \mathrm{C}$ & $1.328 \mathrm{bcde}$ & $1.588 \mathrm{abcd}$ & $1.458 \mathrm{~A}$ & $1.349 \mathrm{bc}$ & $1.680 \mathrm{ab}$ & $1.514 \mathrm{~B}$ \\
\hline Mean & $0.247 \mathrm{~B}$ & $0.379 \mathrm{~A}$ & & $0.246 \mathrm{~B}$ & $0.396 \mathrm{~A}$ & & $1.246 \mathrm{~B}$ & $1.782 \mathrm{~A}$ & & $1.354 \mathrm{~B}$ & $1.628 \mathrm{~A}$ & \\
\hline
\end{tabular}

Notes: $M_{1}, M_{2}, M_{3}, M_{4}$ and $M_{5}$ are mineral nutrients (reference treatment ' $R$ T'), farmyard manure (FYM), vermicompost (VC), Chicken manure (CM) and biogas compost $(\mathrm{BC})$ respectively. $A_{0}$ and $A_{1}$ are none and mycorrhiza respectively.

Relative values of weight per head given by VC, seasons. Such positive effect of VAM occurred only where $\mathrm{CM}, \mathrm{BC}$ and FYM as compared with that given by the organic manuring was given; under conditions of mineral mineral source RT were 95.6, 93.8, 91.9 and $90.7 \%$ fertilization, there was no difference between the VAM respectively (averages of the two seasons). This pattern and no VAM treatments. The pattern of response with occurred particularly under conditions of VAM. The main regard to the total yield was rather similar to that of the effect of VAM shows that the mycorrhiza caused an early yield. average increase of $5.2 \%$ for the early yield over the two

Table 4. Effect of mineral nutrients and organic manures with and without mycorrhiza on artichoke yield

\begin{tabular}{|c|c|c|c|c|c|c|c|c|c|c|c|c|}
\hline \multirow{3}{*}{$\begin{array}{l}\text { Organic } \\
\text { manuring } \\
\text { (M) }\end{array}$} & \multicolumn{12}{|c|}{ Arbuscular mycorrhiza (A) } \\
\hline & \multicolumn{3}{|c|}{ First season } & \multicolumn{3}{|c|}{ Second season } & \multicolumn{3}{|c|}{ First season } & \multicolumn{3}{|c|}{ Second season } \\
\hline & A0 & A1 & Mean & A0 & A1 & Mean & A0 & A1 & Mean & A0 & A1 & Mean \\
\hline & \multicolumn{6}{|c|}{ Early yield $\left(\right.$ kg plant $\left.^{-1}\right)$} & \multicolumn{6}{|c|}{ Total yield $\left(\mathrm{kg} \mathrm{plant}^{-1}\right)$} \\
\hline $\mathrm{M}_{1}$ & $1.451 \mathrm{ab}$ & $1.459 \mathrm{a}$ & $1.455 \mathrm{~A}$ & $1.852 \mathrm{ab}$ & $2.068 \mathrm{a}$ & $1.960 \mathrm{~A}$ & 6.339ab & $6.534 \mathrm{a}$ & $6.436 \mathrm{~A}$ & $6.462 \mathrm{ab}$ & $7.091 \mathrm{a}$ & $6.777 \mathrm{~A}$ \\
\hline $\mathrm{M}_{2}$ & $761 \mathrm{i}$ & $1.336 \mathrm{~b}$ & $1.048 \mathrm{~B}$ & $1.108 \mathrm{~g}$ & $1.848 \mathrm{abc}$ & $1.478 \mathrm{~B}$ & $3.579 \mathrm{~g}$ & $5.189 \mathrm{~cd}$ & $384 \mathrm{C}$ & $3.820 \mathrm{e}$ & $5.554 \mathrm{c}$ & $4.687 \mathrm{C}$ \\
\hline $\mathrm{M}_{3}$ & $1.009 \mathrm{f}$ & $1.127 \mathrm{def}$ & $1.068 \mathrm{~B}$ & $1.442 \mathrm{defg}$ & $1.593 \mathrm{bcdef}$ & $1.517 \mathrm{~B}$ & $4.595 \mathrm{de}$ & $5.772 \mathrm{bc}$ & $183 \mathrm{~B}$ & $4.743 \mathrm{~d}$ & $6.260 \mathrm{bc}$ & $5.502 \mathrm{~B}$ \\
\hline $\mathrm{M}_{4}$ & 373ghi & 1.134 & $1.0035 \mathrm{~b}$ & $1.431 \mathrm{efg}$ & 1.751abcde & $1.591 \mathrm{~B}$ & 4.222 ef & 5.93 & $5.080 \mathrm{~B}$ & $9 \mathrm{de}$ & $6.102 \mathrm{bc}$ & $5.061 \mathrm{BC}$ \\
\hline $\mathrm{M}_{5}$ & & 1.021 & $0.904 \mathrm{C}$ & $1.311 \mathrm{fg}$ & 1.471 cdefg & $1.391 \mathrm{~B}$ & $3.926 \mathrm{fg}$ & $4.583 \mathrm{de}$ & $4.255 \mathrm{C}$ & 3.75 & $5.761 b c$ & $4.756 \mathrm{C}$ \\
\hline \multirow[t]{3}{*}{ Mean } & & 1.215 & & $1.428 \mathrm{~B}$ & $1.746 \mathrm{~A}$ & & $4.532 \mathrm{~B}$ & $5.603 \mathrm{~A}$ & & $4.559 \mathrm{~B}$ & $6.153 \mathrm{~A}$ & \\
\hline & \multicolumn{12}{|c|}{ Weight head $^{-1}(\mathrm{~g})$} \\
\hline & & & Early & yield & & & & & Late & yield & & \\
\hline $\mathrm{M}_{1}$ & & & $282.6 \mathrm{~A}$ & $285.2 \mathrm{abc}$ & $290.8 \mathrm{a}$ & $288.0 \mathrm{~A}$ & $259.5 \mathrm{a}$ & $260.6 \mathrm{a}$ & $260.1 \mathrm{~A}$ & $245.7 \mathrm{ab}$ & $249.2 \mathrm{a}$ & $247.5 \mathrm{~A}$ \\
\hline $\mathrm{M}_{2}$ & & & 269.0 & $271.2 \mathrm{f}$ & $276.9 \mathrm{def}$ & & $224.7 \mathrm{e}$ & $245.1 \mathrm{bcd}$ & $234.9 \mathrm{C}$ & $3 \mathrm{~d}$ & 230. & $225.7 \mathrm{~B}$ \\
\hline $\mathrm{M}_{3}$ & $1.6 \mathrm{c}$ & $281.7 \mathrm{a}$ & $276.7 \mathrm{~B}$ & $277.9 \mathrm{def}$ & $287.8 \mathrm{ab}$ & $282.9 \mathrm{AB}$ & $243.7 \mathrm{~cd}$ & $256.8 \mathrm{ab}$ & $250.3 \mathrm{AB}$ & $230.3 \mathrm{~cd}$ & $238.9 \mathrm{abc}$ & $234.6 \mathrm{~B}$ \\
\hline $\mathrm{M}_{4}$ & $268.3 \mathrm{~d}$ & $277.0 \mathrm{~b}$ & $272.7 \mathrm{C}$ & $276.1 \mathrm{ef}$ & $283.7 \mathrm{bcd}$ & $279.9 \mathrm{BC}$ & $236.1 \mathrm{de}$ & $250.2 a b c$ & $243.2 \mathrm{BC}$ & $223.3 \mathrm{~d}$ & $242.1 \mathrm{abc}$ & $232.7 \mathrm{~B}$ \\
\hline $\mathrm{M}_{5}$ & & & $272.3 \mathrm{C}$ & & 278.9 cde & $276.9 \mathrm{CD}$ & $226.7 \mathrm{e}$ & $248.5 \mathrm{abc}$ & $237.6 \mathrm{C}$ & $224.7 \mathrm{~d}$ & $233.3 \mathrm{bcd}$ & $229.0 \mathrm{~B}$ \\
\hline \multirow[t]{3}{*}{ Mean } & & $277.8 \mathrm{~A}$ & & $277.1 \mathrm{~B}$ & $283.6 \mathrm{~A}$ & & $238.1 \mathrm{~B}$ & $252.2 \mathrm{~A}$ & & $229.1 \mathrm{~B}$ & $238.7 \mathrm{~A}$ & \\
\hline & \multicolumn{12}{|c|}{ Weight of edible part head ${ }^{-1}(\mathrm{~g})$} \\
\hline & \multicolumn{6}{|c|}{ Early yield } & \multicolumn{6}{|c|}{ Late yield } \\
\hline $\mathrm{M}_{1}$ & $71 \mathrm{ab}$ & & $83.59 \mathrm{~A}$ & $82.69 a b c$ & $91.68 \mathrm{a}$ & $87.18 \mathrm{~A}$ & $69.26 \mathrm{a}$ & & $69.97 \mathrm{~A}$ & $74.22 \mathrm{ab}$ & $78.80 \mathrm{a}$ & $76.51 \mathrm{~A}$ \\
\hline $\mathrm{M}_{2}$ & & & $68.09 \mathrm{C}$ & & & & $53.81 \mathrm{e}$ & & $58.40 \mathrm{C}$ & $61.33 \mathrm{~d}$ & $69.21 \mathrm{bc}$ & $65.27 \mathrm{C}$ \\
\hline $\mathrm{M}_{3}$ & $67.74 \mathrm{cde}$ & $78.61 \mathrm{~b}$ & $73.17 \mathrm{~B}$ & $75.03 \mathrm{c}$ & $86.80 \mathrm{ab}$ & $80.91 \mathrm{~A}$ & $62.72 \mathrm{~cd}$ & $67.75 \mathrm{ab}$ & $65.23 \mathrm{~B}$ & $71.22 b c$ & $74.24 \mathrm{ab}$ & $72.73 \mathrm{AB}$ \\
\hline $\mathrm{M}_{4}$ & $68.10 \mathrm{cde}$ & $70.04 \mathrm{~cd}$ & $69.07 \mathrm{C}$ & $76.84 \mathrm{c}$ & $80.60 \mathrm{bc}$ & & $60.73 \mathrm{~d}$ & $65.39 \mathrm{bc}$ & $63.06 \mathrm{~B}$ & $65.10 \mathrm{~cd}$ & $75.92 \mathrm{ab}$ & $70.51 \mathrm{BC}$ \\
\hline $\mathrm{M}_{5}$ & $66.03 \mathrm{de}$ & $71.88 \mathrm{c}$ & $68.95 \mathrm{C}$ & $73.38 \mathrm{c}$ & $80.20 \mathrm{bc}$ & $76.79 \mathrm{~B}$ & $54.14 \mathrm{e}$ & $63.95 \mathrm{~cd}$ & $59.04 \mathrm{C}$ & $66.93 \mathrm{~cd}$ & $71.32 b c$ & $69.12 \mathrm{BC}$ \\
\hline Mean & $69.78 \mathrm{~B}$ & 75.37 A & & $76.29 \mathrm{~B}$ & $83.83 \mathrm{~A}$ & & $60.13 \mathrm{~B}$ & $66.15 \mathrm{~A}$ & & $67.76 \mathrm{~B}$ & $73.90 \mathrm{~A}$ & \\
\hline
\end{tabular}

\section{*See notes of Table 3 for designation of treatments}

\section{Weight of edible part per head}

The highest weight of edible part per head (Table 4) was given by RT+VAM. The lowest was given by FYM in season 1 and by CM in season 2 for early yield. For the late yield the lowest was given by FYM in both seasons. For the early yield, the highest value surpassed the lowest by $31.3 \%$ for season 1 and $24.9 \%$ for season 2 . Comparable percentages for the late yield were 31.5 for season 1 and 
$28.5 \%$ for season 2 . The VC source gave the highest edible part per head among the organic sources, followed by $\mathrm{CM}$ then FYM and BC gave the lowest. This was particularly true where VAM was applied. Over the two seasons, VAM caused an average increase of $9.0 \%$ for the early yield and 9.0 for the late yield. Higher positive effect of VAM occurred only where organic manuring was given since no such significant effect was obtained under conditions of mineral fertilization

Fiber and inulin contents in head:

The highest fibers and inulin were due to RT (Table 5). The lowest fiber content was given by VC or BC. For the early yield, the highest value surpassed the lowest by $14.3 \%$ for season 1 and $26.8 \%$ for season 2 . The pattern of inulin content followed that of the fiber content. Application of VAM caused greater contents of fiber as well as inulin with average increase of 38.7 and $31.4 \%$ for seasons 1 and 2 respectively in early yield. In the late yield VAM caused lower increases of 6.3 and $5.4 \%$ in seasons 1 and 2 , respectively

Table 5. Effect of mineral nutrients and organic manures with and without mycorrhiza on contents of fibers and inulin in artichoke (Cynara scolymus, L.)

\begin{tabular}{|c|c|c|c|c|c|c|c|c|c|c|c|c|}
\hline \multirow{3}{*}{$\begin{array}{l}\text { Organic } \\
\text { manuring } \\
\text { (M) }\end{array}$} & \multicolumn{12}{|c|}{ Arbuscular mycorrhiza (A) } \\
\hline & \multicolumn{3}{|c|}{ First season } & \multicolumn{3}{|c|}{ Second season } & \multicolumn{3}{|c|}{ First season } & \multicolumn{3}{|c|}{ Second season } \\
\hline & A0 & A1 & Mean & A0 & A1 & Mean & A0 & A1 & Mean & A0 & A1 & Mean \\
\hline & \multicolumn{12}{|c|}{ Fibers content $\left(\mathrm{g} \mathrm{kg}^{-1} \mathrm{DW}\right)$} \\
\hline & \multicolumn{6}{|c|}{ Early yield } & \multicolumn{6}{|c|}{ Late yield } \\
\hline $\mathrm{M}_{1}$ & $2.20 \mathrm{a}$ & $2.19 \mathrm{a}$ & $2.20 \mathrm{~A}$ & $2.29 \mathrm{a}$ & $2.25 \mathrm{a}$ & $2.27 \mathrm{~A}$ & $2.11 \mathrm{a}$ & $2.31 \mathrm{a}$ & $2.21 \mathrm{AB}$ & $2.15 \mathrm{a}$ & $2.28 \mathrm{a}$ & $2.21 \mathrm{~A}$ \\
\hline $\mathrm{M}_{2}$ & $2.16 \mathrm{ab}$ & $2.15 \mathrm{ab}$ & $2.16 \mathrm{AB}$ & $2.21 \mathrm{ab}$ & $2.18 \mathrm{ab}$ & $2.20 \mathrm{~A}$ & $2.24 \mathrm{a}$ & $2.28 \mathrm{a}$ & $2.26 \mathrm{AB}$ & $2.26 \mathrm{a}$ & $2.26 \mathrm{a}$ & $2.26 \mathrm{~A}$ \\
\hline $\mathrm{M}_{3}$ & $1.84 \mathrm{de}$ & $1.86 \mathrm{de}$ & $1.85 \mathrm{~B}$ & $1.80 \mathrm{~d}$ & $1.78 \mathrm{~d}$ & $1.79 \mathrm{~B}$ & $2.23 \mathrm{a}$ & $2.12 \mathrm{a}$ & $2.18 \mathrm{~A}$ & $2.22 \mathrm{a}$ & $2.14 b$ & $2.18 \mathrm{~B}$ \\
\hline $\mathrm{M}_{4}$ & $1.93 \mathrm{~cd}$ & $1.94 \mathrm{~cd}$ & $1.94 \mathrm{~B}$ & $1.97 \mathrm{c}$ & $2.01 \mathrm{c}$ & $1.99 \mathrm{~B}$ & $2.19 \mathrm{a}$ & $2.24 \mathrm{a}$ & $2.22 \mathrm{AB}$ & $2.21 \mathrm{a}$ & $2.22 \mathrm{a}$ & $2.22 \mathrm{~A}$ \\
\hline $\mathrm{M}_{5}$ & $1.80 \mathrm{e}$ & $2.04 \mathrm{bc}$ & $1.92 \mathrm{~B}$ & $1.81 \mathrm{~d}$ & $2.09 \mathrm{bc}$ & $1.95 \mathrm{~B}$ & $2.20 \mathrm{a}$ & $2.24 \mathrm{a}$ & $2.22 \mathrm{~B}$ & $2.23 \mathrm{a}$ & $2.20 \mathrm{a}$ & $2.21 \mathrm{~A}$ \\
\hline \multirow[t]{3}{*}{ Mean } & $1.99 \mathrm{~B}$ & $2.03 \mathrm{~A}$ & & $2.02 \mathrm{~B}$ & $2.06 \mathrm{~A}$ & & $2.19 \mathrm{~A}$ & $2.24 \mathrm{~A}$ & & $2.21 \mathrm{~A}$ & $2.22 \mathrm{~A}$ & \\
\hline & \multicolumn{12}{|c|}{ Inulin content $\left(\mathrm{g} \mathrm{kg}^{-1} \mathrm{DW}\right)$} \\
\hline & \multicolumn{6}{|c|}{ Early yield } & \multicolumn{6}{|c|}{ Late yield } \\
\hline $\mathrm{M}_{1}$ & $3.18 \mathrm{c}$ & $4.34 \mathrm{~b}$ & $3.76 \mathrm{~B}$ & $3.24 \mathrm{de}$ & $4.22 \mathrm{c}$ & $3.73 \mathrm{D}$ & $2.44 \mathrm{c}$ & $2.51 \mathrm{bc}$ & $2.48 \mathrm{C}$ & $2.47 \mathrm{bc}$ & $2.58 \mathrm{ab}$ & $2.53 \mathrm{~A}$ \\
\hline $\mathrm{M}_{2}$ & $3.01 \mathrm{c}$ & $3.04 \mathrm{c}$ & $3.03 \mathrm{C}$ & $3.12 \mathrm{e}$ & $3.15 \mathrm{e}$ & $3.13 \mathrm{~A}$ & $2.21 \mathrm{e}$ & $2.28 \mathrm{de}$ & $2.25 \mathrm{AB}$ & $2.23 \mathrm{e}$ & $2.30 \mathrm{de}$ & $2.27 \mathrm{~A}$ \\
\hline $\mathrm{M}_{3}$ & $3.04 \mathrm{c}$ & $5.38 \mathrm{a}$ & $4.21 \mathrm{~A}$ & $3.35 \mathrm{~d}$ & $5.44 \mathrm{a}$ & $4.40 \mathrm{~B}$ & $2.34 d$ & $2.68 \mathrm{a}$ & $2.51 \mathrm{~A}$ & $2.36 \mathrm{~cd}$ & $2.65 \mathrm{a}$ & $2.51 \mathrm{~A}$ \\
\hline $\mathrm{M}_{4}$ & $3.21 \mathrm{c}$ & $4.34 \mathrm{~b}$ & $3.78 \mathrm{~B}$ & $3.33 \mathrm{~d}$ & $4.43 \mathrm{~b}$ & $3.88 \mathrm{~B}$ & $2.54 \mathrm{~b}$ & $2.58 \mathrm{~b}$ & $2.56 \mathrm{~A}$ & $2.56 \mathrm{ab}$ & $2.60 \mathrm{a}$ & $2.58 \mathrm{~A}$ \\
\hline $\mathrm{M}_{5}$ & $3.21 \mathrm{c}$ & $4.28 \mathrm{~b}$ & $3.75 \mathrm{~B}$ & $3.35 \mathrm{~d}$ & $4.33 \mathrm{bc}$ & $3.84 \mathrm{C}$ & $2.44 \mathrm{c}$ & $2.67 \mathrm{a}$ & $2.56 \mathrm{~B}$ & $2.47 \mathrm{bc}$ & $2.64 \mathrm{a}$ & $2.56 \mathrm{~A}$ \\
\hline Mean & $3.10 \mathrm{~B}$ & $4.30 \mathrm{~A}$ & & $3.28 \mathrm{~B}$ & $4.31 \mathrm{~A}$ & & $2.39 \mathrm{~A}$ & $2.54 \mathrm{~A}$ & & $2.42 \mathrm{~B}$ & $2.55 \mathrm{~A}$ & \\
\hline
\end{tabular}

*See notes of Table 3 for designation of treatments

\section{DISCUSSION}

Application of the mineral fertilizer shows superiority in improving the nutritional status of artichoke as well as the growth parameters and yield components over the organic sources. This probably indicates that the readily soluble nutrients are still more preferable for artichoke use than the slow release organic sources in the clayey soils. Generally, the investigated soils are low in their organic matter content. Thus, application of organic amendments probably raised their content in soil of organic matter and this might in turn improve soil-water retention (Abdelhafez et al., 2017), enrich soils with nutrients and increase the availability of non-labile forms of soil nutrients (Farid et al., 2014 and 2018). Moreover, these amendments stimulate the activities of soil microorganisms (Sheng Mao, 2006) which in turn increase phytohormones, and; therefore, affect positively plant growth. Results obtained herein revealed that vermicompost is more preferable than the other organic sources for improving the growth and yield components of artichoke. This might take place because of its high content of nutrients as well as plant growth promoting substances (Bhattacharya and Chattopadhyay, 2002). Also, its low C:N ratio (Table, 2) guarantee rapid decomposition in soil while minimizes the competition between the microorganisms of organic matter and plant roots on the uptake of the available nutrients (Rai, 2006).
The favorable effect of VAM on increasing vegetative growth, NPK content in leaves, early and total yield beside of improving the quality of heads of artichoke is mainly attributed to its ability to increase the solubility of soil phosphorus; while, on the other hand, increases the surface area of absorption of soil nutrients by plant roots (Mohamed et al., 2019), consequently produces activation energy to be utilized in metabolites and building the cells, as well as development of the plant (Rai, 2006). The obtained results are in accordance with those reported by Mohsen (2011) on garlic plants, Shams (2014) and Abdel Nabi et al. (2017) on artichoke as they found that early and total yield were enhanced by application of VAM to the soil, as compared with the un-inoculation plants.

Also, the superiority of Mycorrhiza may be due to symbiosis through greater and faster root structure development as well as through numerous mechanisms such as greater water absorption and a higher resistance to both parasite attacks on the root structure and different types of stress (Ezz El-Din et al., 2010; Mohamed et al., 2019). These effect are in good accordance with those of Shams (2014) on artichoke, and Gmaa (2015) on tomato.

\section{REFERENCES}

A.O.A.C, (2000).Official Methods of Analysis, 13th Ed. Association of Official Agriculture Chemists, Washington, D.C, USA. 
Abdel Nabi, H. M. A., Doklega, S. M. A. and Qwaider, A. F. S. (2017). Effect of Organic, Bio Fertilization and Foliar Spraying Treatments on Artichoke. J. Plant Production, Mansoura Univ. 8(5): 559 - 567

Abdelhafez, A. A., Abbas, H. H., Abd-El-Aal, R. S., Kandil, N. F., Li, J. and Mahmoud, W. (2012). Environmental and Health Impacts of Successive Mineral Fertilization in Egypt. Clean Soil Air Water, 40: 356-363. https://doi.org/10.1002 /clen. 201100151

Abdelhafez, A.A., Abbas, M.H.H., Li, J. (2017). Biochar: The Black Diamond for Soil Sustainability, Contamination Control and Agricultural Production. In. (ed) Engineering Applications of Biochar, InTech Open pp 7-27 http://dx. doi.org/ 10.5772/intechopen.68803

Abdelhafez, A.A., Abbas, M.H.H., Attia, T.M.S., El Bably, W., Mahrous, S.E. (2018). Mineralization of organic carbon and nitrogen in semi-arid soils under organic and inorganic fertilization, Environmental Technology \& Innovation, 9: 243253, https://doi.org/10.1016/j.eti.2017.12.011.

Ahmed, N.A.M., Abbas, H.H., El-Ashry, S.M., Abbas, M.H.H. (2013). The Feasibility of Using Unconventional Fertilizers on $\mathrm{P}$ Availability in Soil. Egypt J. Soil Sci. 53 (1): 55-65. https:// doi.org / 10.21608/ejss.2013.140

Alshaal T. et al. (2019). Soil Health and Its Biology. In: ElRamady H., Alshaal T., Bakr N., Elbana T., Mohamed E., Belal AA. (eds) The Soils of Egypt. World Soils Book Series. Springer, Cham, pp 175185. https://doi.org/10.1007/978-3-319-95516-2 10

Ardakani, M. R, D. Mazaheri, S. Mafakheri and A. Moghaddam (2011). Absorption efficiency of N, P, $\mathrm{K}$ through triple inoculation of wheat (Triticum aestivum L.) by Azospirillum brasilense, Streptomyces sp., Glomus intraradices and manure application. Physiol Mol Biol Plants, 17 (2):181192. https://doi.org/10.1007/s12298-011-0065-7

Bassouny, M.A., Abbas, M.H.H. (2019). Role of biochar in managing the irrigation water requirements of maize plants: the pyramid model signifying the soil hydro-physical and environmental markers. Egypt. J. Soil. Sci. 59 (2): 99-115. https://doi.org/ 10.21608/EJSS.2019.9990.1252

Barno, A., Ondanje, B., Ngwiri, J. (2011). Dynamics of horticultural export to Europian union market: challenges and opportunities in sub-saharan Africa. SHS Acta Horticulturae 911: 61-72. https://doi.org/ 10.17660/ActaHortic.2011.911.7

Bhattacharya,S.S. and Chattopadhyay, G.N. (2002). Increasing bioavailability of phosphorus from fly ash through vermicomposting. J. Environ. Quality 31(6): 2116-2119. https://doi.org/10.2134/ jeq2002 .2116

Black, C.A., (1982). Methods of Soil Analysis. Part 2. American Society of Agronomy, INC., publisher, Madison, Wisconsin, USA.

Duggan, T., Jones, P., (2016). Lettuce (Lactuca sativa 'Webb's Wonderful') shoot and root growth in different grades of compost and vermicomposted compost. Acta Hortic. 1146: 33-40 https://doi.org/ 10.17660/ActaHortic.2016.1146.4

El-Katkat, M.B.O. (1992). Studies on dissolving phosphorus by some microorganism. M.Sc. Thesis, Fac. Agric, Al-Azhar Univ, Egypt.

Ezz El-Din A. A, Aziz, E. E., Hendawy, S.F. and Omer, E.A. (2010). Impact of phosphorus nutrition and number of cuttings on growth, yield and active constituents of artichoke. International Journal of Academic Research, 2 (4): 240-244.
Farid, I.M., Abbas, M.H.H., Beheiry, G. G.S. and Elcossy, S.A.E. (2014). Implications of organic amendments and tillage of a sandy soil on its physical properties and C-sequestration as well as its productivity of wheat and maize grown thereon. Egypt J. Soil. Sci. 54(2): 177-194. https://doi.org/ 10.21608 /ejss. 2014.132

Farid, I., Abbas, M.H.H., El-Ghozoli, A. (2018). Implications of humic, fulvic and K-humate extracted from each of compost and biogas manures as well as their teas on Faba bean plants grown on a Typic Torripsamment soil and emissions of -soil CO2. Egypt J. Soil Sci 53 (3): 275-289. https://doi.org/10.21608/ejss.2018.3386.1168

Fratianni, F., Tucci, M., Palma, M.D., Pepe, R., Nazzaro, F. (2007). Polyphenolic composition in different parts of some cultivars of globe artichoke (Cynara cardunculus var. scolymus (L.) Fiori), Food Chemistry, 104 (3): 1282-1286, https://doi.org/ 10.1016/j.foodchem.2007.01.044.

Frutos, M.J., Ruiz-Cano, D., Valero-Cases, E., Zamora, S. and Pérez-Llamas, F. (2019). Chapter 3.2 - Artichoke (Cynara scolymus L.), In: Seyed Mohammad Nabavi, Ana Sanches Silva (eds.) Nonvitamin and Nonmineral Nutritional Supplements, Academic Press, pp 135-138. https://doi.org/10.1016/B978-012-812491-8.00018-7.

Gmaa, S.S. (2015). Effect of organic and biofertilization on tomato production. Intern. J. of Adv. Res., 3(10): 1799-1805.

Gomez, K. A. and Gomez A. A. (1984). Statistical Procedures in Agricultural Research. John Wiley and Sons, New York, 2nd ed.

Horton, S. and Mannar, M.G.V. (2018). Chapter 31 Economics of Food Fortification, In: M.G. Venkatesh Mannar, Richard F. Hurrell, Food Fortification in a Globalized World, Academic Press, 299-304, https://doi.org/10.1016/B978-0-12-802861-2.00031-6

Jackson, M.L. (1973). Soil Chemical Analysis. Prentice-Hall India, Private limited, New Delhi.

Duhan, J.S., Kumar, R., Kumar, N., Kaur, P., Nehra, K. and Duhan, S. (2017). Nanotechnology: The new perspective in precision agriculture, Biotechnology Reports15: 11-23, https://doi.org/ 10.1016/ j.btre 2017.03.002.

Kumar, M.S., Rajiv, P., Rajeshwari, S. and Venckatesh, R. (2015). Spectroscopic analysis of vermicompost for determination of nutritional quality, Spectrochimica Acta Part A: Molecular and Biomolecular Spectroscopy 135: 252-255, https://doi.org/ 10.1016 / j.saa.2014.07.011.

Lampkin, N. (1990). Organic farming. Farming press books. Ipswich. United Kingdom.

Lattanzio, V., Kroon, P.A., Linsalata, V., Cardinali, A. (2009). Globe artichoke: A functional food and source of nutraceutical ingredients, Journal of Functional Foods, 1 (2): 131-144, https://doi.org/ 10.1016/j.jff.2009.01.002.

Liorach, R, Espin, J. C., Tomas, F. A. and Ferreres, F. (2002). Atichoke (Cynara scolymus) byproduct as a potential source of Health promoting antioxidant phenolics. J. Agri. and Food Chem. 50: 3458-3464. http://dx.doi.org/10.1021/jf0200570

Mohamed, I., Eid, K.E., Abbas, M.H.H., Salem, A.A., Ahmed, N., Ali, M., Shah, G.M. and Fang, C. (2019). Use of plant growth promoting Rhizobacteria (PGPR) and mycorrhizae to improve the growth and nutrient utilization of common bean in a soil infected with white rot fungi. Ecotoxicology and Environmental Safety, 171: 539-548. https://doi.org/ 10.1016/ j.ecoenv. 2018.12. 100 
Mohsen, A.A.M., El-Mansi, A.A.A., Khalil, M.A.I. and Arisha, H.M.E. (2011). Influence of mycorrhiza and mineral phosphorus application on growth yield and quality of garlic plants grown in sandy soil. Egypt. J. of Appl. Sci., 26 (10): 269-280.

Mupambwa, H.A. and Mnkeni, P.N.S. (2018). Optimizing the vermicomposting of organic wastes amended with inorganic materials for production of nutrientrich organic fertilizers: a review. Environ Sci Pollut Res 25: 10577-10595. https://doi.org/ 10.1007 /s11356-018-1328-4

Nest, T.V., Ruysschaert, G., Vandecasteele, B., Houot, S., Baken, S., Smolders, E., Cougnon, M., Reheul, D. and Merckx, R. (2016). The long term use of farmyard manure and compost: Effects on $\mathrm{P}$ availability, orthophosphate sorption strength and $\mathrm{P}$ leaching, Agriculture, Ecosystems \& Environment 216: 23-33, ttps://doi.org/ 10.1016/ j.agee. 2015.09.009.

Rai, M. K. (2006). Microbial biofertilizers, Haworth press, Inc. 10 Alce Street, Pinghamton, NY 13904-1580.

Rouphael, Y., Colla, G., Graziani, G., Ritieni, A., Cardarelli, M. and Pascale, S.D. (2017). Phenolic composition, antioxidant activity and mineral profile in two seed-propagated artichoke cultivars as affected by microbial inoculants and planting time, Food Chemistry, 234: 10-19, https://doi.org/ 10.1016/j.foodchem.2017.04.175.

Shams, A. S. (2014). Effect of silver nano-particles and mycorrhizae symbiosis on the development of artichoke. International Journal of Agricultural Science and Research. 4(5): 27-36.

Sheng Mao, Y., Feng Min, L., Tian Wen, G., Jian Guo, W., Bing Ling, S. and Shao Ling, J. (2006). Effect of long term fertilization on soil productivity and nitrate accumulation in Gansu Oasis, China. Agric. Sci. in China, 5 (1): 57-67 (C.F. computer Search).

SPSS 15.0 Command Syntax Reference (2006).SPSS Inc, Chicago III, USA.

Sradnick, A., Oltmanns, M. and Raupp, J. (2018) Microbial biomass and activity down the soil profile after long-term addition of farmyard manure to a sandy soil. Org. Agr. 8: 29-38. https://doi.org/ 10.1007/s13165-016-0170-6
Uddin, J., A.H.M. Solaiman and M. Hasanuzzaman (2009). Plant characteristics and yield of Kohlabi (Brassica oleracea var. gongylodes) as affected by different organic manures. J. Hort. Sci. Ornament. Plants 1: 1-4

Vadeboncoeur, M.A., Ouimette, A.P. and Hobbie, E.A. (2015). Mycorrhizal roots in a temperate forest take up organic nitrogen from ${ }^{13} \mathrm{C}$ - and $15 \mathrm{~N}$-labeled organic matter. Plant Soil 397: 303-315. https://doi.org/10.1007/s11104-015-2623-1

Vernon, G. (1999). Sustainable vegetable production. Ithca Inc. New York. USA.

Verzeaux, J., Hirel, B., Dubois, F., Lea, P. J. and Tétu, T. (2017). Agricultural practices to improve nitrogen use efficiency through the use of arbuscular mycorrhizae: Basic and agronomic aspects,Plant Science, 264: 48-56, https://doi.org/ 10.1016/ j.plantsci.2017.08.004.

Wakindiki I.I.C., Malobane M.E. and Nciizah A.D. (2019). Integrating Biofertilizers with Conservation Agriculture Can Enhance Its Capacity to Mitigate Climate Change: Examples from Southern Africa. In: Leal Filho W., Leal-Arcas R. (eds) University Initiatives in Climate Change Mitigation and Adaptation. Springer, Cham, pp 277-289. https://doi.org/10.1007/978-3-319-89590-1 16

Wiedenhoeft, A. C. (2006). Plant Nutrition.Chelsea House Publishers, US.

Winton, A.L. and Winton, K.B. (1958).The analysis of foods. John Wiley and Sons, Inc. London, PP: 857.

Zhang, L., Shi, N., Fan, J., Wang, F., George, T. S. and Feng, G. (2018). Arbuscular mycorrhizal fungi stimulate organic phosphate mobilization associated with changing bacterial community structure under field conditions. Environ Microbiol, 20: 2639-2651. doi:10.1111/1462-2920.14289

\section{تقييم كفاعة السماد العضوي مقابل التسميد المعني مع أو بلون الميكوريزا على المحصول وخصائص الخرشوف

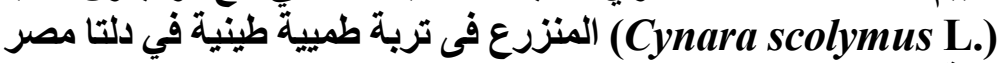 قـم التنمية مختار مرسمى المتواصلة للبيئة وإدارة مشروعاتها ـ معهد الاراسات والبحوث البيئية ـ جامعة مدينة السادات ـ مصر}

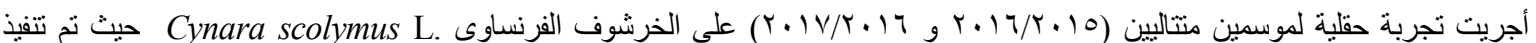

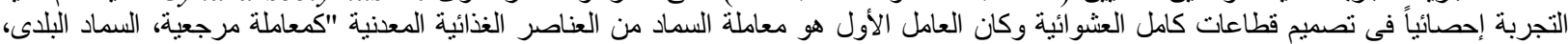

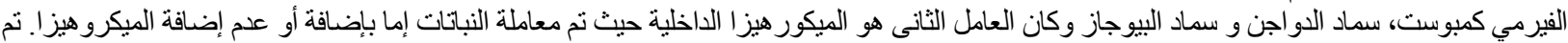

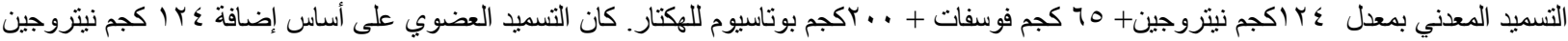

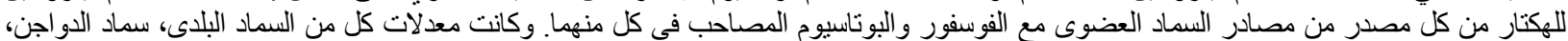

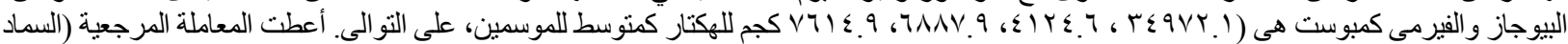

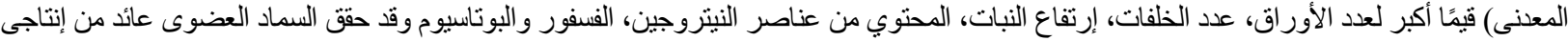

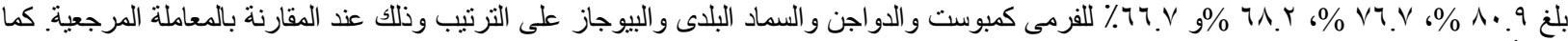

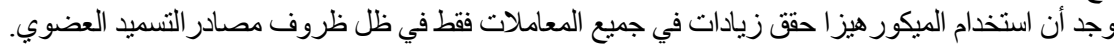

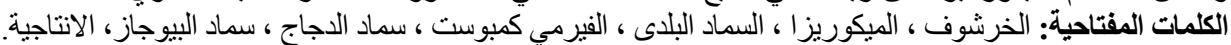

